\title{
Los proyectos de integración del arte en educación (arts integration) y la mejora de la calidad docente a través de un estudio de caso
}

\author{
Margarida Llevadot González; Anna Pagès Santacana*
}

Resumen. A través de un estudio de caso en profundidad, el proyecto Arte y Escuela del Centro de Artes Contemporáneas de Vic (ACVIC), se analiza un proyecto de integración del arte en educación que hace visible un alto nivel de colaboración y reconocimiento mutuo entre profesionales del arte y la educación favoreciendo nuevas propuestas, metodologías y formas de evaluar. En las conclusiones se definen los referentes e indicadores para orientar una propuesta de proyectos de integración del arte en educación entre instituciones educativas y culturales en beneficio de la calidad docente.

Palabras clave: Integración del arte en educación; arte y educación; innovación pedagógica; educación en las instituciones culturales; trabajo por proyectos; comunidad de aprendizaje.

PROJETOS DE INTEGRAÇÃO ARTÍSTICA NA EDUCAÇÃO (INTEGRAÇÃO ARTÍSTICA) E A MELHORIA DA QUALIDADE DO ENSINO ATRAVÉS DE UM ESTUDO DE CASO

Resumo. Este trabalho pretende analisar, mediante um estudo de caso em profundidade do projeto Arte e Escola do Centro de Artes Contemporâneas de Vic (ACVIC), um projeto de integração da arte na educação que torna visível um alto nível de colaboração e reconhecimento mútuo entre os profissionais da arte e da educação, favorecendo novas propostas, metodologias e formas de avaliar. Nas conclusões, são definidos os referentes e indicadores para orientar uma proposta de projetos de integração da arte na educação entre instituições educacionais e culturais em prol da qualidade do ensino.

Palavras-chave: Integração da arte na educação; arte e educação; inovação pedagógica; educação nas instituições culturais; trabalho por projetos; comunidade de aprendizagem.

ARTS INTEGRATION PROJECTS AND THE IMPROVEMENT OF TEACHING QUALITY THROUGH A CASE STUDY

Abstract. Through an in-depth case study - the Art and School project of ACVIC - we have analyzed a project of integration of art in formal education that brings to light a high level of collaboration and mutual recognition between professionals of art and education and favors new proposals, methodologies and assessment methods. In the conclusions, we have defined the quality conditions of an Arts Integration project between educational and cultural institutions for the benefit of teaching quality.

Keywords: Arts integration; art and education; pedagogical innovation; education in cultural institutions; learning community.

Universidad Ramón Llull, España. 


\section{LOS PROYECTOS DE INTEGRACIÓN DEL ARTE EN EDUCACIÓN (ARTS INTEGRATION)}

A partir de la década de los ochenta, en Europa y Estados Unidos, el arte en la escuela adquirió una mayor presencia. Se consideró que el arte daba más oportunidades a los niños al combinar inteligencia y sensibilidad, reflexión y emoción (Gadamer, 1984; Eisner, 1987, Kerlan,2007). Sin embargo, durante largo tiempo, las políticas culturales y educativas habían aislado al arte de la educación escolar y viceversa, generando barreras en el proceso de aprendizaje y su dimensión creativa, convirtiendo a la escuela en receptora de actividades estandarizadas (Pagès, 2011) con poco margen de participación y aprendizaje por parte de alumnos y maestros.

Desde hace una década, la tendencia de las instituciones artísticoculturales (museos, centros artísticos etc.) ha sido reorientar su tarea educativa centrándose en el sujeto que aprende y planteando propuestas nuevas, más centradas en el proceso creativo del aprendizaje y en los modos de relación con el conocimiento en un contexto de diálogo y colaboración en red. El cambio de paradigma supone entender la institución artístico-cultural como un espacio situado en el circuito de aprendizaje y de transformación social. Este enfoque ha fomentado la investigación de otros canales de participación: nuevos proyectos de investigación, creación y experimentación, nuevas metodologías y prácticas colaborativas (Parramon, 2012; Macaya, 2013).

El concepto de integración del arte en educación (Arts Integration) tiene sus raíces en el constructivismo y en los movimientos progresistas de carácter globalizador de la Escuela Nueva. Aunque la nueva modalidad de Arts Integration es algo más que un trabajo por proyectos, se inspira en la idea inicial de Dewey y Kilpatrick en torno a los proyectos de trabajo o Project based learning. En Estados Unidos, uno de los centros pioneros que promueve la integración del arte en las escuelas desde una institución cultural y artística es el John F. Kennedy Center for the Performing Arts (KC), en Washington, el centro cultural nacional para las artes escénicas de primer nivel y de más envergadura. Uno de los programas que impulsa la institución es el Changing Education Through the Arts program (CETA program -"Programa de transformación de la educación a través de las Artes"-). Los líderes del programa CETA definen el concepto Arts Integration como una metodología de enseñanza en la que los estudiantes demuestran el conocimiento aprendido a través de una forma artística comprometiéndose en el proceso creativo y conectando con otras áreas de conocimiento a través del desarrollo gradual de los objetivos previstos de las dos áreas. Las razones principales del interés de los docentes por las prácticas de integración del arte tienen que ver con el hecho de que se adaptan a la forma de aprender de los estudiantes, desarrollan una mayor comprensión y curiosidad intelectual, así como más 
tolerancia, confianza, motivación y autoexigencia- y al mismo tiempo son prácticas que renuevan los profesionales de la educación, dándoles más energía y satisfacción (Chand O’Neal, 2014).

EI KC promueve parejas de trabajo entre profesionales que proceden tanto del mundo educativo como del artístico-cultural, intercambiando ideas, metodologías y nuevas maneras de evaluar. Este impulso de renovación procede del exterior del sistema educativo reglado y se inserta en la escuela a través de los docentes, con la colaboración estrecha de artistas o asesores artísticos, constituyendo parejas de trabajo entre profesionales y también entre instituciones educativas y culturales.

A partir de la idea comunitaria de un conjunto de prácticas de Arts Integration, se han ido impulsando proyectos que modifican una tendencia política de dispersión y pocas sinergias aprovechando la colaboración entre profesionales del mundo educativo y cultural y las instituciones y administraciones que les apoyan. Se trata de un conjunto de prácticas de revitalización de la tarea educativa, generando espacios de participación, de investigación y de producción, así como de apoyo y de visibilidad en las escuelas, haciendo posible el trabajo colectivo entre profesionales e instituciones del mundo educativo y cultural, con el fin de transformar la experiencia y el espacio social (Parramon, 2009). En estos proyectos la creatividad es una herramienta importante de transformación, ya que permite desarrollar la capacidad de crear nuevos imaginarios, y también hacer del arte y de la cultura una experiencia formativa cotidiana, del día a día. Valorar más el proceso artístico que su producto final y hacer del arte una experiencia cotidiana significa considerar que la sensibilidad y la imaginación no son exclusivas de las disciplinas artísticas: están presentes en todas las formas de conocimiento (Collelldemont, 2001). Esto implica ampliar la concepción de la cultura y del arte y conducirla más allá, como una práctica compartida con otros que facilita la transformación de la sociedad, al impulsar nuevas ideas en personas que piensan creando al mismo tiempo nuevos espacios de debate, de relación y de convivencia.

¿Cuáles son las condiciones idóneas para una óptima hibridación entre los ámbitos educativo (maestros) y artístico (asesores) en los procesos colaborativos entre instituciones educativas y culturales? ¿De qué manera la práctica educativa se transforma después de haber participado en un proyecto de integración del arte en educación? La hipótesis que fundamenta la presente investigación se puede definir en los siguientes términos: la propia experiencia artística de los impulsores y asesores de este tipo de proyectos constituye un factor clave de calidad en la concepción y desarrollo de un proyecto de integración del arte en educación. Con el fin de determinar cuáles son las condiciones idóneas para un buen desempeño de esta experiencia 
de «integración» entre arte y educación, se requiere explorar el proceso del proyecto en el momento de su implementación, analizando de qué forma prioriza la calidad y equidad en las prácticas educativas propuestas. El enfoque metodológico más adecuado para este objetivo es la realización de un estudio de caso en profundidad.

Así pues, los objetivos de la presente investigación son analizar, a través de un estudio de caso, -el proyecto Arte y Escuela del Centro de Artes Contemporáneas de Vic (ACVIC)- Ios siguientes factores: el papel del asesor artístico, la función de las parejas profesionales (asesor -maestro) y las actividades comunes que se llevan a cabo, identificando y describiendo los elementos clave del proceso de transformación y mejora de la experiencia escolar.

\section{UN ESTUDIO DE CASO SINGULAR DE INTEGRACIÓN DEL ARTE EN EDUCACIÓN: ARTE Y ESCUELA DEL CENTRO DE ARTES CONTEMPORÁNEAS DE VIC (ACVIC)}

Los criterios básicos para la elección del estudio de caso de Arte y Escuela del Centro de Artes Contemporáneas de Vic (ACVIC) son los siguientes: a. Presentar una perspectiva integradora entre arte y pedagogía por parte de un equipamiento cultural público, entendiendo que el hecho educativo va más allá de la escuela y que el hecho cultural y artístico no es exclusivo de los museos o centros de arte. b. Incluir un trabajo en equipo entre profesionales del mundo educativo y cultural en un proyecto común con liderazgo compartido, que lleva a cabo proyectos para que profesores y artistas dialoguen y utilicen las artes para estimular la creatividad y la imaginación. c. Ser un equipamiento cultural público y un proyecto que favorecen el trabajo en red entre centros educativos poniendo en relación a muchos maestros en un mismo curso y año tras año. d. Constituir un proyecto que promueve nuevas formas de relación en estrecha colaboración entre las políticas culturales y las educativas e. Ofrecer un modelo de liderazgo que facilita al investigador una fácil entrada al trabajo de campo.

ACVIC surge de la colaboración de una asociación de artistas y creadores- de H. Asociación para las Artes Contemporáneas fundada en 1991-, y de dos administraciones, la local y la autonómica. El centro cultural pertenece a la Red de Centros Territoriales de Artes Visuales de Cataluña. 
En 2010, el director y el equipo de gestión de $\mathrm{H}$ definieron cuatro programas. Dentro del programa LAB,Arte y Escuela pretende potenciar nuevas metodologías de aprendizaje basadas en la creatividad a partir de lo que ya está haciendo la escuela. El proyecto se estructura en cuatro fases: a) formación o asesoramiento por parte del equipo de asesores b) jornadas de intercambio de experiencias c) exposición en el centro cultural d) maleta pedagógica. Arte y Escuela + Luz (2014-2015), ha sido el tema de la cuarta edición del proyecto Arte y escuela donde se han llevado a cabo el estudio de caso donde han participado 30 centros educativos, se han realizado 35 proyectos y ha implicado a 3.200 alumnos. A partir de un eje temático común -en este caso la luz- los asesores de arte y los maestros compartieron e intercambiaron ideas y recursos mediante el vínculo entre prácticas artísticas contemporáneas y educativas durante todo un curso escolar.

\section{ETAPAS DEL ESTUDIO, RECOGIDA DEL MATERIAL ETNOGRÁFICO Y PROCEDIMIENTO DE ANÁLISIS}

El estudio de caso para esta investigación se basa en un enfoque holístico, global y relacional (Morin, 2000), inductivo y emergente -ya que se construyen nuevos significados a partir de las informaciones que se van obteniendo -y un enfoque ideográfico para interpretar la particularidad y la trama de las relaciones. Se trata de aproximarse al trabajo de integración del arte en la educación, para ver el proceso que sigue y cómo va tomando forma, una "forma-formante" en términos de Maffesoli, que configura el cuerpo social y define la sociedad al mismo tiempo (Maffesoli, 1997).

El estudio de caso tiene tres etapas diferenciadas que se han ido definiendo en el transcurso de la investigación: el papel del asesor, las parejas profesionales de trabajo y las actividades comunes. El procedimiento de recogida de material etnográfico relevante se ha estructurado desde las siguientes acciones de campo: a) observación participante in situ; b) entrevistas con componente biográfico y entrevistas colectivas en un formato cercano a la conversación; c) cuestionario escrito de tres preguntas abiertas; d) fuentes documentales y e) diario de campo de tipo etnográfico. En el marco de la investigación etnográfica, el procedimiento de análisis tiene como objetivo extraer e interpretar el sentido de los datos e intentar reconstruir una cultura determinada, acercarse al significado que los sujetos participantes en los contextos estudiados atribuyen a las realidades o fenómenos.

Etapa 1: El papel del asesor artístico. El análisis del contenido está estructurado a partir de la observación participante de las cinco reuniones de trabajo del equipo de asesores artísticos recogida en el diario de campo 
y complementada con las actas de cada reunión y con las seis entrevistas a informantes clave que asistían a las reuniones. Las entrevistas complementan, sobre todo, la tercera reunión del equipo dedicada exclusivamente a discutir cuál es la tarea del asesor. La entrevista permite describir e interpretar aspectos de la realidad desde la perspectiva de los actores. Siguiendo a Velasco y Díaz de Rada (2009), la entrevista constituye un elemento fundamental en el proceso de realización del trabajo de campo para conocer el discurso del protagonista del estudio. Se trata de comprender la idea del proyecto desde lo que los informantes narran sobre su experiencia profesional y fijándose en las coincidencias de sus discursos. Con esta intención, las entrevistas orales individuales han tenido un cierto componente biográfico; no han seguido un guion definido o estructurado por el investigador, sino que se pidió a los asesores que contaran su experiencia con el proyecto Arte y Escuela para así complementar y comprender mejor la información obtenida a partir de la observación participante de las reuniones. Los tres informantes clave entrevistados individualmente y por este orden son: una profesora de Universidad, la coordinadora del Centro de Recursos Pedagógicos y la coordinadora de los proyectos educativos del Centro de Arte Contemporáneo. Las entrevistas colectivas en forma de conversación han sido, por este orden, con el director de ACVIC y una profesora de Universidad; dos artistas y profesores de la Escuela de Arte Superior de Diseño de Vic que comparten los asesoramientos; y dos asesores del colectivo de artistas Morir de frío que han sido asesores y comisarios de la exposición Arte y Escuela + Luz en ACVIC. Se ha realizado una transcripción de las entrevistas para sistematizar, vaciar y analizar los datos obtenidos y se han establecido comparaciones entre las categorías de análisis de la observación participante de las reuniones de los asesores y las categorías de análisis de las entrevistas a los asesores.

Etapa 2: Las parejas profesionales de trabajo. Para abordar metodológicamente el análisis de las parejas de trabajo se ha llevado a cabo una observación participante de las tutorías en la escuela de la maestra y dos conversaciones con ella donde se le pidió que relate sus vivencias con el proyecto Arte y Escuela de dos maneras diferentes: en la primera entrevista, al final del proyecto Arte y Escuela + Luz (2014-2015), se pidió a la maestra que relatara su experiencia con el proyecto de manera libre, resaltando lo que considerara oportuno. Se otorgó un valor concreto al relato hecho historia, a la persona que crea y configura su propia historicidad, a la construcción de un hilo narrativo propio (Ferrarrotti, 2011). La segunda conversación, un año más tarde, en 2016, se solicitó a la maestra que relatara, a través de fotografías que previamente ella misma había seleccionado, los trabajos de sus alumnos de la edición de la luz y de la edición siguiente Ilamada Arte y Escuela + Vacío (2015-2016). En tercer lugar, se analizaron los textos del 
catálogo de la exposición Arte y Escuela + Luz escrito por las maestras de las distintas escuelas para analizar cómo sintetizaban por escrito las actividades llevadas a cabo y cuáles destacaban.

Etapa 3. Las actividades comunes. En la observación participante de las cuatro actividades del proyecto se anotó en el diario de campo de tipo etnográfico cuáles fueron los aspectos organizativos, qué personas intervinieron, qué acciones se desarrollaron, qué dijeron, cómo se relacionaban, etc. En segundo lugar, se estableció una conversación con los dos comisarios de la exposición para ver qué tratamiento hacían los artistas del trabajo realizado por los escolares. Se trataba de analizar el paso del trabajo educativo escolar al trabajo artístico: como se realizaba y en qué condiciones. En tercer lugar, con el fin de facilitar la recogida de datos, se hizo llegar un cuestionario con tres preguntas abiertas por escrito -vía correo electrónico- a los maestros que participaron en la edición del proyecto Arte y Escuela+luz a través del Centro de Recursos Pedagógicos de Osona. Para tener una visión general sobre qué dicen los maestros de los beneficios que les aporta el proyecto se les plantearon tres preguntas sobre los motivos para involucrarse y participar en el proyecto, así como también cuáles fueron los valores añadidos de la experiencia.

A continuación, se presenta un cuadro sintético de la recogida del material etnográfico de las acciones e instrumentos, de los temas de investigación, de las personas implicadas y del lugar donde se ha llevado a cabo el trabajo de campo:

\section{TABLA 1}

Cuadro sintético de la recogida del material etnográfico del proyecto Arte y Escuela + luz

\begin{tabular}{|l|l|l|l|}
\hline \multicolumn{1}{|c|}{ Acciones e instrumentos } & Etapas y temes & \multicolumn{1}{|c|}{ Personas } & \multicolumn{1}{|c|}{ Lugares } \\
\hline Entrevistas individuales & $\begin{array}{l}\text { El papel del } \\
\text { asesor }\end{array}$ & $\begin{array}{l}2 \text { coordinadores y } \\
\text { una asesora }\end{array}$ & $\begin{array}{l}\text { CRP Osona, } \\
\text { ACVIC, Universitat } \\
\text { de Vic }\end{array}$ \\
\hline Entrevistas colectivas & $\begin{array}{l}\text { El papel del } \\
\text { asesor }\end{array}$ & 6 asesores & $\begin{array}{l}\text { Escuela de Arte, } \\
\text { Vic, ACVIC i } \\
\text { Barcelona }\end{array}$ \\
\hline $\begin{array}{l}\text { Observación participante a } \\
\text { las reuniones del equipo de } \\
\text { asesores }\end{array}$ & $\begin{array}{l}\text { El papel del } \\
\text { asesor }\end{array}$ & $\begin{array}{l}\text { Grupo de 15 } \\
\text { asesores }\end{array}$ & ACVIC \\
\hline $\begin{array}{l}\text { Análisis de les actas de les } \\
\text { reuniones }\end{array}$ & $\begin{array}{l}\text { El papel del } \\
\text { asesor }\end{array}$ & Investigadora & Barcelona \\
\hline $\begin{array}{l}\text { Observación participante } \\
\text { asesoramiento maestra -asesora }\end{array}$ & $\begin{array}{l}\text { Parejas de } \\
\text { trabajo }\end{array}$ & Asesora-maestra & Escuela en Osona \\
\hline
\end{tabular}




\begin{tabular}{|c|c|c|c|}
\hline Acciones e instrumentos & Etapas y temes & Personas & Lugares \\
\hline Entrevistas con la maestra & $\begin{array}{l}\text { Parejas de } \\
\text { trabajo }\end{array}$ & Maestra & Escuela en Osona \\
\hline $\begin{array}{l}\text { Observación participante en las } \\
\text { jornadas de estudiantes }\end{array}$ & $\begin{array}{l}\text { Actividades } \\
\text { comunes }\end{array}$ & 10 escuelas & Universidad de Vic \\
\hline $\begin{array}{l}\text { Observación participante en la } \\
\text { jornada de profesorado }\end{array}$ & $\begin{array}{l}\text { Actividades } \\
\text { comunes }\end{array}$ & 6 escuela & Escuela en Vic \\
\hline Cuestionario a los maestros & $\begin{array}{l}\text { Actividades } \\
\text { comunes }\end{array}$ & 26 maestros & $\begin{array}{l}\text { Vic, comarca } \\
\text { de Osona y } \\
\text { adyacentes }\end{array}$ \\
\hline $\begin{array}{l}\text { Observación participante en } \\
\text { la presentación de la maleta } \\
\text { pedagógica }\end{array}$ & $\begin{array}{l}\text { Actividades } \\
\text { comunes }\end{array}$ & $\begin{array}{l}4 \text { asesores } \\
\text { responsables } \\
\text { (y una trentena } \\
\text { de persones } \\
\text { entre maestros y } \\
\text { asesores) }\end{array}$ & ACVIC \\
\hline Visita a la exposición & $\begin{array}{l}\text { Actividades } \\
\text { comunes }\end{array}$ & Investigadora & ACVIC \\
\hline Entrevista a los comisarios & $\begin{array}{l}\text { Actividades } \\
\text { comunes }\end{array}$ & $\begin{array}{l}2 \text { asesores } \\
\text {-comisarios }\end{array}$ & Barcelona \\
\hline $\begin{array}{l}\text { Análisis del catálogo de la } \\
\text { exposición }\end{array}$ & $\begin{array}{l}\text { Actividades } \\
\text { comunes }\end{array}$ & Investigadora & Barcelona \\
\hline Diario de camp etnográfico & $\begin{array}{l}\text { Durante el } \\
\text { trabajo de } \\
\text { campo }\end{array}$ & $\begin{array}{l}\text { Elaboración propia } \\
\text { de la investigadora }\end{array}$ & Barcelona y Vic \\
\hline
\end{tabular}

Fuente: elaboración propia

\section{EL PAPEL DEL ASESOR DE UN PROYECTO DE ARTS INTEGRATION: ELEMENTOS CRÍTICOS}

El asesoramiento artístico del proyecto Arte y Escuela consiste en un acompañamiento en activo a maestros de los centros educativos de educación infantil, primaria o secundaria participantes con el fin de implementar el proyecto Arte y Escuela. El enfoque del asesoramiento parte del reconocimiento de la acción creativa de la educación y de los maestros. Este ha sido un punto clave para elegir el estudio de caso del ACVIC. El asesor y el maestro trabajan conjuntamente pero cada uno tiene unas funciones y responsabilidades diferentes. Aunque ambos investigan nuevas metodologías, será el maestro quien creará el proyecto con sus estudiantes, quien desarrollará el proyecto en el aula con el apoyo externo del asesor. Se confirmó la hipótesis inicial: la función del asesor artístico constituye una de las condiciones de calidad en la implementación y el desarrollo de proyectos de integración de arte-educación. 
Durante la investigación se identificaron algunos elementos clave para precisar de qué manera los asesores artísticos integran el arte en la educación y deducir algunas consecuencias en relación con la hipótesis inicial. Entre estos elementos críticos es relevante subrayar los siguientes: a. el trabajo en equipo entre asesores b. la función mediadora del arte y las parejas profesionales de trabajo (asesor-maestro) y c la red de relaciones con la comunidad educativa

a. Trabajo en equipo entre asesores: El proyecto Arte y Escuela integra el arte en la educación gracias a un trabajo en equipo donde los asesores tienen funciones formativas y también toman decisiones en la organización del proyecto, vinculando así la acción educativa con el trabajo planificado. Los obstáculos que encuentran los asesores en contacto con la escuelas se convierten en oportunidades para pensar y crear un proyecto común. El equipo de asesores artísticos reconvierte estas dificultades en oportunidades para definir mejor los tempos del proyecto, construyéndolo y difundiendo de manera colaborativa.

\section{b. La función mediadora del arte y las parejas de trabajo asesor-} maestro: Los asesores escuchan, complementan, valoran a los maestros con una metodología no fijada a priori y abierta a múltiples formas y perspectivas. No se trata de un tipo de asesoramiento concluyente sino relacional, intermitente, incierto y arriesgado. Pretende transmitir que el arte es una manera de hablar entre personas, un lenguaje, un punto de partida, una idea con múltiples interpretaciones. Para ello se aporta experiencia técnica, se facilitan recursos y documentación y se dan ideas de actividades concretas que los maestros Ilevan a la práctica. Los asesores dejan espacio y tiempo para que el maestro adopte las propuestas a su manera. No ayudan al maestro, sino que más bien comparten ideas, dan confianza, valoran y apoderan. Maestros y asesores aprenden unos de otros, de lo emergente que está pasando y no sólo de lo sabido y reconocido. En el proceso de asesoramiento se constata una correspondencia entre la experiencia con el arte de los asesores y la relación pedagógica que establecen con el maestro mientras Ilevan a cabo el proyecto. Cuando los asesores hablan de su asesoramiento hacen referencia al arte como un elemento transversal que integra la incertidumbre, el azar, el error, la sutileza y la multiplicidad de puntos de vista. Cuando el arte se inserta o se integra en las prácticas educativas tiene una función mediadora: pone la atención en los vínculos, las relaciones y el afecto a través de compartir saberes, experiencias y lenguajes y de estar dispuesto a explorar nuevas posibilidades y metodologías.

c. Red de relaciones con la comunidad educativa. Desde esta perspectiva y vivencia del arte es desde donde los asesores hacen posible llevar a cabo un proyecto de colaboración con la escuela que estrecha los vínculos entre 
el profesorado y los asesores artísticos externos, revitaliza y renueva trabajo educativo y se expande e involucra las administraciones y las instituciones educativas y culturales que le dan soporte tanto a nivel local como autonómico.

\section{LAS PAREJAS DE TRABAJO ASESOR-DOCENTE. MICROANÁLISIS DE LA TRANSICIÓN ARTE-EDUCACIÓN}

Las parejas de trabajo del proyecto Arte y Escuela están formadas por un asesor artístico (en algunos casos dos) y por un maestro (algunas veces implica un ciclo, o toda la escuela). ¿Cuál es la función de las parejas de trabajo arte-educación? Se ha analizado, en primer lugar, las respuestas de la asesora artística a los requerimientos de la maestra y; en segundo lugar, de qué manera una maestra incorpora las ideas y propuestas artísticas en sus prácticas. A partir de la conversación que establecen las dos profesionales se ha ido deduciendo que aporta de nuevo esta relación. Se trataba de hacer un microanálisis para ver en detalle cómo transita el proyecto de lo educativo a lo artístico y viceversa. La proximidad con las protagonistas permitió desvelar los detalles que hacen visible el tránsito del proyecto de lo educativo a lo artístico, si ha habido transferencia, analizando como la maestra ha preparado y realizado las actividades. La mejor manera de ver qué ha aprendido la maestra es a través de sus explicaciones sobre el proyecto y las reflexiones sobre los trabajos de sus alumnos en dos ediciones diferentes. En cada una de las ediciones del proyecto Arte y Escuela la maestra ha tenido asesores artísticos diferentes. No interesó tanto analizar los trabajos realizados por estudiantes, sino focalizar la mirada sobre los aprendizajes de la maestra, de qué manera lo cuenta y hasta qué punto ha cambiado su manera de enfocar las prácticas.

A lo largo del análisis se ha comprobado cuál era la demanda de la maestra: pide a la asesora que la propuesta de proyecto para Arte y Escuela sea un proyecto significativo y que se pueda relacionar con algún proyecto que quieran trabajar en el aula. Al mismo tiempo, la asesora solicitaba que el proyecto tuviera intencionalidad artística. Entre las dos buscaban que la transdisciplinariedad del proyecto se hiciera de manera sencilla y acotada. La asesora mostraba a la maestra otros ejemplos de maestros y otros artistas y animaba a combinar y relacionar distintos referentes -con el apoyo presencial de otra persona con más experiencia- proporcionando algunos materiales y recursos para concretar su propuesta de una manera diferente. La asesora puso énfasis en la importancia de mostrar el proceso creativo del proyecto a la hora de exponer la producción final en el ACVIC y señaló también otras formas de representación. Aunque en el proceso es donde está todo el peso del proyecto, la asesora velaba, también, porque la representación final en 
el ACVIC tuviera una calidad y dignificara lo que se hace en las escuelas. La maestra repetía la experiencia vivida en sus clases dando más voz y participación a los alumnos, haciéndoles pensar en otros ejemplos en vez de copiarlos, sopesando diferentes puntos de vista. La asesora invertía la lógica instrumental convirtiéndola en un abordaje más intuitivo, relacionando más bien la forma, el contenido y el contexto, es decir, la globalidad del proyecto. De manera esquemática se resume en el siguiente cuadro:

\section{TABLA 2}

La transición arte-educación

\begin{tabular}{|l|l|}
\hline \multicolumn{2}{|c|}{ Confluencias entre las demandas de la asesora y las de la maestra } \\
\hline La asesora artística subraya: & La maestra requiere: \\
\hline La intención artística del proyecto & Un proyecto significativo \\
\hline Propuesta acotada y transdisciplinar & La concreción de actividades \\
\hline $\begin{array}{l}\text { Pensar (no copiar) los referentes: obras } \\
\text { de artistas, proyectos de otras escuelas }\end{array}$ & $\begin{array}{l}\text { Integrar el proyecto interdisciplinario interno de } \\
\text { la escuela con el proyecto Arte y Escuela en todo }\end{array}$ \\
\hline $\begin{array}{l}\text { Relacionar forma, contenido y } \\
\text { contexto a través de invertir la lógica } \\
\text { instrumental por la lógica de les } \\
\text { percepciones e intuiciones }\end{array}$ & Incorporar nuevas ideas y materiales \\
\hline $\begin{array}{l}\text { Mostrar el proceso } \\
\text { Presencia de los niños como creadores }\end{array}$ & Incorporar los puntos de vista de los niños. \\
\hline
\end{tabular}

Fuente: Elaboración propia

¿Qué función tienen las parejas de trabajo arte-educación? ¿Qué beneficios comporta para los maestros la integración del arte en el trabajo educativo? ¿De qué manera un proyecto transita del hecho educativo al hecho artístico? A lo largo del estudio de caso se comprobó como la conversación entre la maestra y la asesora será el medio para ir definiendo el proyecto. La función de las parejas de trabajo es hacer posible la transición de los proyectos educativos interdisciplinarios de las escuelas a proyectos interdisciplinarios con intencionalidad artística. El asesoramiento artístico proporciona a la maestra la oportunidad, la confianza y las herramientas para que encuentre su manera de hacer y configure identidad y sensibilidad estética que mejore la calidad de los proyectos iniciales que le propone. La maestra necesita un interlocutor con quien poder compartir las ideas iniciales, tener referentes para poder actuar de otra manera y volver a consensuar lo pensado de nuevo para acabar concretando como se muestra el proyecto realizado por la escuela en el centro de arte. La asesora y la maestra co-crean junto el proyecto con responsabilidades diferentes. La primera enseña con su ejemplo a co-crear 
para que después la maestra también lo haga con sus estudiantes. No puede haber integración sin una transición, sin un interlocutor que guíe el proceso. La figura del asesor artístico es capital para integrar el arte en la educación.

El proyecto tiene una intencionalidad artística cuando las nuevas ideas del asesor se integran bien en las ideas pensadas por los maestros y al mismo tiempo las ideas de los maestros se modifican y adoptan un nuevo formato. Esta nueva manera de hacer consiste en relacionar el contexto, el contenido y la forma para que el todo tenga sentido. Para conseguirlo, se invierte la lógica instrumental para la lógica de las percepciones e intuiciones. Se muestra el proceso seguido y compartido donde quedan reflejados los diferentes puntos de vista de los niños. Por lo tanto, para la transición de los proyectos es necesario haberlos pensado mucho, matizarlos, compartirlos en el espacio natural donde suceden. El proceso de transición es un proceso lento que se produce por contagio y necesita tiempo. Implica un cambio gradual y pone el énfasis en el proceso y la experiencia vivida más que en la producción cultural resultante. Los proyectos se van desarrollando teniendo en cuenta las experiencias de la maestra, el proceso seguido así y la reflexión compartida entre maestra y asesora.

\section{LAS ACTIVIDADES COMUNES Y ELEMENTOS CRÍTICOS PARA LA INTEGRACIÓN DEL ARTE EN EDUCACIÓN.}

En la tercera etapa del estudio de caso se analizaron las diferentes actividades comunes del proyecto Arte y Escuela que son el resultado de la colaboración entre diferentes profesionales del mundo educativo y artístico: a) las Jornadas de estudiantes en la universidad b) la Jornada del profesorado c) la exposición y d) la maleta pedagógica.

a) La asistencia a las Jornadas de estudiantes ha servido para observar cómo se organizan las jornadas; también entender por qué se hacen en la universidad y no en la escuela. Igualmente se ha reflexionado sobre la presentación de los trabajos por parte de los estudiantes. ¿Qué valor añadido tiene la universidad en la presentación de los trabajos escolares? Es importante subrayar que los estudiantes y sus escuelas muestren su proyecto bien documentado, que se vea bien el proceso seguido y así poder explicarlo mejor a los demás. Al mismo tiempo, es esencial el diálogo para valorar críticamente los proyectos que han hecho otros compañeros de edades similares y también recibir las valoraciones de los asesores artísticos. Se trata de preparar con claridad la exposición para ejemplificar el proceso realizado, compartir el pro- 
yecto con otros compañeros de escuelas diferentes y recibir una valoración de los asesores artísticos externos a la escuela. Estos son tres elementos críticos para el buen funcionamiento de las Jornadas de estudiantes a la universidad.

b) ¿Qué beneficios aporta el proyecto a los docentes y qué dicen los maestros en las Jornadas del profesorado sobre su experiencia en uno de los subgrupos? Se ha llevado a cabo una observación de algunos subgrupos escogidos al azar. Cada grupo de maestros de cada escuela ha expuesto los criterios de elaboración y puesta en práctica de sus proyectos: cuál fue su reto, los detalles del proceso seguido, las reacciones de los estudiantes, los trabajos resultantes, etc. Las dos jornadas del proyecto Arte y Escuela permiten a maestros y estudiantes poder mostrar y compartir lo que hacen en la escuela y aprender de lo que hacen el resto de los compañeros de otras escuelas que trabajan sobre un mismo tema y, al mismo tiempo, recibir una valoración por parte de los asesores artísticos. Esta exposición requiere una mayor exigencia al presentar los trabajos por parte de maestros y de alumnos. Al mismo tiempo, a través del proyecto Arte y Escuela, los asesores artísticos comprenden las necesidades de la escuela y preparan actividades y recursos más adecuados a sus necesidades.

Para poder tener una visión más amplia sobre qué piensa y cómo ha vivido el proyecto el profesorado, se facilitó un cuestionario con tres preguntas abiertas escritas a todos los maestros participantes. El cuestionario respeta el anonimato y facilita la expresión de ideas. De una muestra de treinta y cinco personas respondieron veintiséis, procedentes de escuelas públicas, concertadas y rurales y profesores de diferentes niveles educativos desde infantil hasta secundaria. Las tres preguntas del cuestionario fueron las siguientes: ¿Cuáles han sido las motivaciones, razones o inquietudes que le han Ilevado a participar en el proyecto Arte y Escuela? ¿Podría describir en qué parte del proyecto hubo un buen entendimiento entre el maestro y el asesor?; ¿Cuáles son los valores añadidos de la experiencia? En la tabla 3 , expondremos los ítems más frecuentes en relación con las tres preguntas formuladas.

¿De qué manera la práctica educativa se transforma después de haber participado en un proyecto como el del ACVIC? La mayoría de los maestros subrayan la actitud de escucha del asesor artístico, la aportación de nuevos referentes y recursos y la posibilidad de consensuar ideas y resolver dudas. El asesor tiene un papel muy relevante, pero no quiere ser el protagonista del proyecto, sino adoptar un papel de interlocutor, de facilitador. La sutileza del asesor es uno de los aspectos que atraviesan este liderazgo artístico. A través de los asesores artísticos, los maestros se sienten autorizados, apoyados y animados a probar nuevas prácticas a través de las posibilidades que ofrece 
el arte. Afirman que el proyecto les permite conocer mejor su alumnado y favorece el pensamiento reflexivo, crítico y creativo de los niños, lo que aumenta su motivación y placer por aprender.

\section{TABLA 3}

\section{Motivaciones, elementos de buen entendimiento interprofesional y valor añadido de la experiencia según los maestros}

\section{Valoración del proyecto de integración del arte en educación según los maestros}

\begin{tabular}{|l|l|}
\hline $\begin{array}{l}\text { Motivaciones que expresan los } \\
\text { maestros para involucrarse en } \\
\text { el proyecto: }\end{array}$ & $\begin{array}{l}\text { Compartir un tema común con otras escuelas e } \\
\text { intercambiar ideas entre maestros y entre alumnos } \\
\text { Fomentar el arte y la creatividad y compensar una carencia } \\
\text { en la escuela } \\
\text { Favorecer la transversalidad entre disciplinas } \\
\text { Formar parte de un proyecto global }\end{array}$ \\
\hline Elementos de buen \\
entendimiento entre maestros y y \\
asesores artísticos & $\begin{array}{l}\text { Escucharse mutuamente } \\
\text { Acompañar al maestro a emocionarse y soñar } \\
\text { Apoyar: No obligar ni presionar } \\
\text { las ideas de las escuelas, el asesor trae ideas y materiales } \\
\text { Intervenir positivamente: Enfocar y conducir } \\
\text { Proporcionar recursos, ideas y tiempo } \\
\text { Dar visiones diferentes }\end{array}$ \\
\hline Valor añadido de la experiencia \\
según los maestros & $\begin{array}{l}\text { Conocer mejor a los alumnos } \\
\text { Compartir el proyecto con otros compañeros } \\
\text { Estimular el aprendizaje con retos que rebasan las tareas } \\
\text { habituales de la escuela }\end{array}$ \\
\hline Fuente:elaboracion propia.
\end{tabular}

Fuente:.elaboración propia.

$c$ y d): ¿Qué tipo de material pedagógico alternativo puede surgir de los proyectos integrados arte-escuela? Los comisarios de la exposición tendrán mucho cuidado de no contaminar la obra hecha e integrarla en un nuevo discurso expositivo, es decir de respetar lo que significa la obra para los niños y, posteriormente, introducirla en la historia que los comisarios quieren explicar y mostrar. Con la maleta, los asesores no dan ideas de entrada sino materiales para explorar y experimentar nuevas maneras de hacer en el aula. Valoran y promueven el arte en la escuela a partir de las experiencias que ya han sido probadas y han tenido más relevancia. A partir de aquí, facilitan materiales para seguir investigando y creando. 


\section{LA CALIDAD Y LA INTEGRACIÓN DEL ARTE EN EDUCACIÓN}

Arte y Escuela del Centro de Arte Contemporáneo de Vic es un caso excepcional por su alto nivel de colaboración y reconocimiento mutuo entre los profesionales del arte y la educación. ¿Cuáles son los elementos de referencia para una buena integración del arte en educación?

\section{La importancia del proceso de un proyecto de integración del Arte} en Educación. Para saber cuáles son las condiciones idóneas para una experiencia de calidad de integración del arte en educación, es necesario seguir el proceso del proyecto en el momento que se lleva a cabo y analizar cómo favorece la calidad y la equidad de las prácticas educativas. En el estudio de caso se ha comprobado que los proyectos no se predeterminan o anticipan de entrada ni a nivel organizativo ni formativo, sino que se van construyendo conjuntamente favoreciendo un auténtico trabajo colaborativo que combina el trabajo riguroso y la invención original.

2. Presencia sostenida del impulso creativo-artístico. El impulso creativo está presente no solo en la puesta en marcha del proyecto, sino también en la gestión y el asesoramiento y las actividades conjuntas. El proceso creativo no depende exclusivamente de la creación artística: está presente en todas las actividades compartidas.

3. Presencia y permanencia de una idea singular. Los impulsores del proyecto y los asesores artísticos trabajan en equipo y comparten decisiones de tipo organizativo y formativo, a partir de una idea singular. Es esta idea singular -la mirada del arte como un proceso complejo, dinamizador de conocimiento y de relaciones - la que permite superar y reconducir los obstáculos de la relación Arte-Educación en oportunidades para crear e innovar, definiendo mejor los tempos del proyecto y difundiendo de manera colaborativa.

4. Las parejas de trabajo dialógicas. Los asesores trasladan esta manera de trabajar en equipo en relación con los maestros en el momento de constituir las parejas de trabajo. En el modo de asesoramiento se constata una correspondencia entre la experiencia con el arte de los asesores y la relación pedagógica que establecen con los maestros mientras Ilevan a cabo el proyecto. Cuando los asesores hablan de su propia práctica hacen referencia al arte como un elemento transversal y mediador que integra la incertidumbre, el azar, el error, la sutileza y la multiplicidad de puntos de vista.

\section{El grado de inserción del arte como experiencia en el ambiente} escolar: Cuando el arte se integra en la actividad de los maestros pone la atención en los vínculos, las relaciones y el afecto a través de compartir saberes, experiencias y lenguajes y de estar dispuesto a explorar nuevas po- 
sibilidades y metodologías. Los asesores complementan a los maestros con una metodología no fijada, aportan experiencia técnica, facilitan recursos y dan ideas de actividades concretas que los maestros Ilevan a la práctica, abriendo un espacio y un tiempo diferentes para que el maestro adopte las propuestas a su manera haciendo visible la capacidad creativa del maestro. En Arte y Escuela el maestro se siente más libre y autorizado a llevar a cabo sus ideas, ver que su práctica y la de sus alumnos ha mejorado, valorarlo y compartir conocimientos y metodologías con otros maestros de otras escuelas.

6. La importancia de la conversación entre maestra y asesora. La conversación entre la maestra y la asesora facilita la transición de los proyectos de integración desde el mundo del arte a la educación. Es una conversación libre, no preparada, donde ambas pueden expresar libremente sus propuestas y dudas. La maestra necesita un interlocutor con quien poder compartir las ideas iniciales, tener referentes para poder actuar de otra manera y consensuar lo pensado de nuevo para acabar concretando como se muestra el proyecto realizado por la escuela.

7. La transición arte-educación. Tal y como se ha indicado anteriormente, la función de las parejas de trabajo maestra-asesora consiste en convertir la transición de los proyectos educativos interdisciplinarios en proyectos interdisciplinarios con intencionalidad artística. Esto sucede cuando las nuevas ideas del asesor se integran bien en las ideas pensadas por los maestros y, al mismo tiempo, las ideas de los maestros se modifican y adoptan un nuevo formato. Esta nueva manera de hacer consiste en relacionar el contexto, el contenido y la forma para que el conjunto tenga sentido. Para conseguirlo, se invierte la lógica instrumental por la lógica de las percepciones e intuiciones La asesora y la maestra co-crean conjuntamente el proyecto con responsabilidades diferentes. La primera enseña con su ejemplo y testimonio para que luego la maestra también lo haga con sus estudiantes. La figura del asesor artístico es capital para integrar el arte en educación.

8. La importancia de las actividades comunes: exponer-se. Las actividades comunes del proyecto Arte y Escuela permiten a maestros y estudiantes poder mostrar, expresar y compartir lo que hacen en la escuela y aprender de lo que hacen el resto de los compañeros de otras escuelas que trabajan sobre un mismo tema y, al mismo tiempo, recibió una valoración por parte de los asesores artísticos. Cuando los asesores valoran la labor realizada en la escuela también se exponen; y este exponerse requiere de una mayor exigencia. Con este tipo de proyecto compartido, los asesores artísticos comprenden las necesidades de la escuela y preparan actividades y recursos más adecuados. El arte se va integrando y difundiendo entre asesores, maestros y estudiantes. 
9. El asesor se sitúa como un interlocutor en lugar de un experto. La mayoría de los maestros remarcan del asesor artístico la actitud de escucha, la aportación de nuevos referentes, puntos de vista y recursos, y la posibilidad de consensuar ideas y resolver dudas. En Arte y Escuela las ideas surgen de los maestros y son acogidas por los asesores externos. El protagonismo recae en los estudiantes y sus maestros; el asesor es un interlocutor externo al sistema educativo con quien poder hablar y les da alas para poder hacer cosas diferentes a las establecidas. El asesor no juzga, no se presenta como un experto que ha de dar consignas precisas, sino como un compañero con quien compartir perspectivas desde una lógica diferente que no coincide con la orientación hacia los resultados característica del sistema escolar. Se inserta una cierta gratuidad en la experiencia de la práctica escolar compartida. Gracias a los asesores artísticos, los maestros se sienten autorizados y animados a probar nuevas maneras de hacer a través de las posibilidades que ofrece el arte. Los maestros afirman en las jornadas y los cuestionarios que el proyecto les hace conocer mejor a sus alumnos y favorece el pensamiento reflexivo, crítico y creativo de los niños porque aumenta su motivación y placer por aprender.

10. La exposición y la maleta pedagógica como herramientas de relación y materiales de apoyo alternativos. ¿Qué tipos de material pedagógico alternativo puede surgir en este entorno? Los comisarios definen el espacio expositivo como algo a construir, no fijado a priori. Se irá construyendo a partir de la relectura de la obra de arte, es decir, del trabajo de los niños, de la historia que cada proyecto ha querido explicar. A la hora de preparar la exposición, los comisarios tendrán mucho cuidado de respetar el mensaje y representación del trabajo de los niños para luego integrarlos en un nuevo discurso expositivo en el Centro de Artes Contemporáneas. La maleta pedagógica es una herramienta más de relación entre el mundo educativo y el cultural-artístico. Este recurso valora y promueve el arte a partir de las experiencias que ya han sido probadas y han tenido más eco en las escuelas. A partir de la valoración de los trabajos de las escuelas, los asesores crean unos materiales abiertos y de calidad, interés y provecho para las escuelas y el centro cultural y, al mismo tiempo, favorecen la sostenibilidad del proyecto.

A continuación, de manera gráfica, se detallan los elementos de registro para identificar e impulsar buenas prácticas de proyectos de integración del arte en educación: 


\section{TABLA 4}

\section{Referentes e indicadores para la integración del arte en educación}

\begin{tabular}{|c|c|}
\hline Referentes & Indicadores \\
\hline La importancia del proceso & $\begin{array}{l}\text { Proyectos no predeterminados ni } \\
\text { estandarizados }\end{array}$ \\
\hline $\begin{array}{l}\text { Presencia sostenida del impulso creativo- } \\
\text { artístico }\end{array}$ & Aprendizaje mutuo \\
\hline Presencia de una idea singular permanente & $\begin{array}{l}\text { La mirada del arte como un proceso } \\
\text { complejo, dinamizador de conocimiento y } \\
\text { de relaciones }\end{array}$ \\
\hline Las parejas de trabajo dialógicas & $\begin{array}{l}\text { Asesoramiento relacional, intermitente, } \\
\text { incierto, arriesgado }\end{array}$ \\
\hline $\begin{array}{l}\text { El grado de inserción del arte como } \\
\text { experiencia Enel ambiente escolar }\end{array}$ & Práctica reflexiva \\
\hline $\begin{array}{l}\text { La importancia de la conversa entre maestra } \\
\text { y asesora }\end{array}$ & Presencia y conversación no pautada, libre \\
\hline La transición arte-educación & $\begin{array}{l}\text { Inversión de la lógica instrumental por la } \\
\text { lógica sensible }\end{array}$ \\
\hline La importancia de les actividades comunes & Exponerse. Compartir y valorar \\
\hline $\begin{array}{l}\text { El asesor se sitúa como interlocutor y no como } \\
\text { experto }\end{array}$ & Atención, cura y sensibilidad \\
\hline $\begin{array}{l}\text { La exposición y la maleta pedagógica y } \\
\text { la importancia de un material de soporte } \\
\text { alternativo }\end{array}$ & Material abierto \\
\hline
\end{tabular}

Fuente: Elaboración propia.

\section{CONCLUSIONES: LA MEJORA DE LA CALIDAD DOCENTE}

En el proyecto Arte y Escuela del ACVIC se ha comprobado que la acción educativa -en contacto con el arte- se convierte en una acción creativa favoreciendo una mayor apreciación, confianza mutua y colaboración entre los profesionales de los mundos educativo y artístico-cultural. El enfoque holístico y al mismo tiempo incierto -característico del mundo del arte- facilita al maestro correr riesgos en su propia práctica en un sistema cada vez más burocratizado y orientado a los resultados. El asesor artístico permite al maestro orientarse desde otra perspectiva y lo autoriza a experimentar de otra forma gracias a esta integración del arte en la práctica educativa. La experiencia escolar resulta finalmente más amable y, al mismo tiempo, más exigente. Asesores, maestros y estudiantes se sienten escuchados, acompa- 
ñados y valorados con las ideas que expresan y de la manera que lo hacen, y lo pueden contrastar con otros compañeros y colegas de la profesión y en diferentes entornos: la escuela, la universidad y el Centro de Artes Contemporáneas. De este modo, el proyecto Arte y Escuela revaloriza y revitaliza las prácticas educativas compartidas. La creatividad y la innovación surgen de la colaboración entre maestros y asesores artísticos. Estos últimos dan, desde fuera de la escuela, pero con una estrecha colaboración con ella, el contexto y el impulso necesarios para que los maestros se atrevan a crear y estén satisfechos.

El proyecto Arte y escuela ofrece una formación continuada a los maestros muy vinculada a sus prácticas. Los asesores colaboran con los maestros y dan un plus de calidad a las propuestas que los maestros Ilevarán a cabo con sus alumnos. Las propuestas que se hacen son nuevas y al mismo tiempo se interrelacionan bien con las dinámicas de la escuela. Además, estas buenas experiencias hacen que se sumen otras escuelas y otros maestros que quizás no están tan acostumbrados al mundo del arte o que necesitan ver que estas experiencias con el arte funcionan. Fuera del sistema escolar pero estrechamente vinculada a él, la institución cultural juega un papel fundamental en la hibridación entre la educación, el arte y la cultura: valora y difunde lo que ya se hace en la escuela, hace hincapié en los procesos, da tiempo para detenerse, pensar, esperar y repetir -a menudo prácticas poco habituales en los currículos escolares apretados o a las políticas y metodologías exclusivamente orientadas a los resultados-. La experiencia con el arte en las prácticas compartidas favorece una mayor porosidad del sistema educativo y cultural y los hace más permeables e integradores de propuestas diversas que se interrelacionan.

Para concluir, se puede afirmar que la integración del arte en educación incrementa el proceso de enculturación y humanización, impulsando relaciones dialógicas colaborativas de confianza y aprendizaje mutuo, donde todos y todas se sienten más escuchados y considerados desplegando mejor sus posibilidades profesionales en el servicio a los demás.

\section{BIBLIOGRAFÍA}

Chand O'Neal, I. (2014). Selected Findings from the John F. Kennedy Center's Arts in Education Research Study: An Impact Evaluation of Arts-Integrated Instruction through the Changing Education through the Arts (CETA) Program. Recuperat de https://artsedge. kennedy-center.org/educators/how-to/arts-integration/ceta-white-paper Washington.

Collelldemont, E. (2006). Els espais i els temps en l'Educació Estètica. Revista Catalana de Pedagogia, 5, 15-32. 
Eisner, E. W. (1987). Procesos cognitivos y currículum. Barcelona, Espanya: Martínez Roca.

Ferrarotti, F. (2011). Las historias de vida como método. Acta Sociológica, 56, 95-119.

Gadamer, H. G. (1984). Verdad y método. Salamanca, Espanya: Sígueme.

Kerlan, A. (2007). L'art pour éduquer. La dimensión esthétique dans le projet de formation postmoderne. Education et sociétés, 19, 83-97.

Macaya, A. (abril de 2013). Els projectes, punt de trobada entre museus i escola. A A. Macaya, A, R. Ricomà i M. Suárez (Presidencia), Arts, educació i interdisciplinarietat. Simpòsium dut a terme a les XI Jornades de Pedagogia de I'Art i Museus, Tarragona, Espanya.

Maffesoli, M. (1997). Elogio de la razón sensible: una visión intuitiva del mundo contemporáneo. Barcelona, Espanya: Paidós

Morin, E. (2000). La mente bien ordenada. Barcelona, Espanya: Seix Barral.

Pagès, A. (2011). Temps de transmissió. Les seves vicissituds avui. Lleida, Espanya: Pagès editors

Parramon, R. (2009). Accions reversibles en procés. Arte, educación, territorio. ACVIC-Eumo, 15-33

Stake, R. E. (2007). Investigación con estudio de casos. Madrid, Espanya: Ediciones Morata.

The Kennedy Center. (1990-2017). Changing Education Through the Arts (CETA): Professional learning for educators and teaching artists. Washington, D.C, USA: The Kennedy Center. Recuperat de http://education.kennedy-center.org//education/ceta/ 